\title{
Pressure Control Design of High-Pressure Fuel Pipe
}

\author{
Shi Wei", Chunyong Wang, Tailin Cen, Yuhua Su
}

College of Teachers and Music, Hezhou University, Hezhou, China

\section{Email address:}

weishi666666@163.com (Shi Wei)

${ }^{*}$ Corresponding author

\section{To cite this article:}

Shi Wei, Chunyong Wang, Tailin Cen, Yuhua Su. Pressure Control Design of High-Pressure Fuel Pipe. American Journal of Applied Mathematics. Vol. 9, No. 4, 2021, pp. 92-103. doi: 10.11648/j.ajam.20210904.11

Received: June 7, 2021; Accepted: June 28, 2021; Published: July 5, 2021

\begin{abstract}
In order to ensure the normal operation of the engine, the stability of the fuel supply system must be ensured. The fuel supply system needs to obtain fuel from the outside, and then pressurized into the high-pressure oil pipe, and then input into the engine cylinder. Therefore, the fuel parameters in the components of the fuel supply system affect each other. In this paper, a mathematical model is established to describe the relationship between the fuel parameters of each part of the fuel supply system, and then the stability of the fuel supply system can be controlled by controlling some parameters under given conditions. We intercept a very short time, analyze the mutual transfer of fuel in the fuel supply system through the micro element method, and establish the differential equations. Because some differential equations are more complex, it is difficult to find the formula solution, so we discretize the differential equations and get a group of recurrence relations. After calculating the initial state and related parameters, the fuel pressure parameters and fuel density parameters of each part of the fuel supply system at each time can be obtained recursively. Because the pressure and density of the fuel can express each other, we focus on the density parameter of the fuel, and do the conversion when the pressure is needed. On the basis of this group of recursive relations, we established an optimization model of fuel pressure control in high-pressure oil pipe: Taking the fuel supply time of one-way valve as decision variables, minimizing the difference between fuel pressure and standard value at each time as optimization objective, taking the practical significance of each physical quantity as constraint condition, and gave a discrete algorithm.
\end{abstract}

Keywords: High-Pressure Fuel Pipe, The Stability of Fuel Supply Pressure, The Micro Element Method, Differential Equations, The Optimization Model

\section{Introduction}

stability of the fuel supply system (Figure 1) must be improved

In order to ensure the normal operation of the engine, the

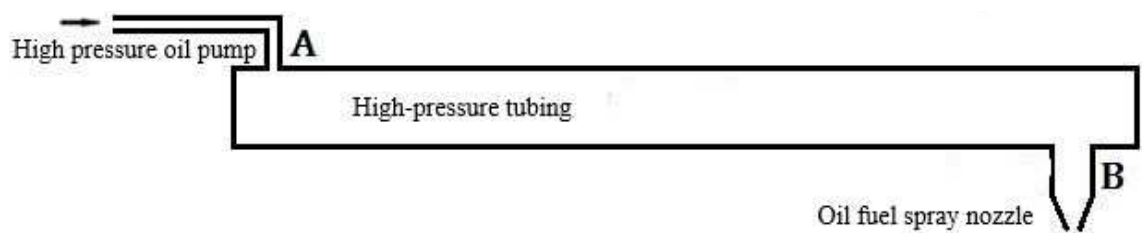

Figure 1. Schematic diagram of fuel supply system.

Question 1: The relevant parameters of high pressure oil pipe, fuel supply port and injection nozzle are given, and it is required to adjust the fuel supply time of each opening of the one-way valve A in order to keep the fuel supply pressure in the high-pressure fuel pipe as stable as possible.
Question 2: The cam is added to drive the plunger to feed oil into the high-pressure fuel pipe. Under the condition that the relevant parameters of the cam and other components are determined, the angular velocity of the cam is obtained to keep the fuel supply pressure as stable as possible. 
Question 3: As shown in Figure 2, a fuel injection nozzle and an one-way pressure reducing valve are added on the basis of problem 2, which is required to determine the angular speed of the cam, the fuel supply law of each nozzle, and the time for the one-way pressure reducing valve to open at one time, so as to maintain the stability of the fuel supply pressure.

In order to better solve the above problems, make some reasonable assumptions:

(1) It is assumed that the conduit connecting the high-pressure fuel pump and the high-pressure fuel pipe is very thin and its volume is negligible.

Reason: the conduit is very thin relative to the oil storage container in the actual production, and its volume can be ignored.

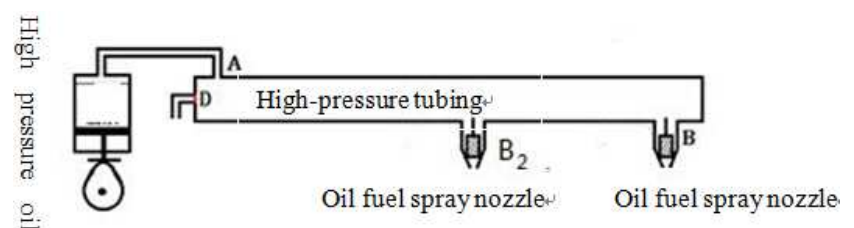

Figure 2. Schematic diagram of high-pressure fuel pipe with pressure reducing valve and two injectors.

(2) It is assumed that the one-way valve A opens and closes instantly, regardless of the gradual opening and closing process of the valve A.

Reason: the gradual opening and closing buffering process of the fuel injection nozzle B has been considered. if the valve A opening and closing buffering process is taken into account, the treatment method is the same; and the cushioning property of the valve $\mathrm{A}$ is not given in the design, so the gradual opening and closing process of the valve $\mathrm{A}$ is not considered.

(3) the beginning of the suction stroke of the engine, the pressure inside the cylinder is negligible.

Reason: at the beginning of the suction stroke of the engine, the piston has squeezed out most of the gas in the cylinder, and the pressure inside the cylinder is very small, so it can be ignored as a simplified model.

(4) It is assumed that the angular velocity of the cam rotation is constant.

Reason: In the process of mechanical transmission, the uniform rotation of the cam is easy to design.

In order to make the engine run normally, the stability of the fuel supply system must be ensured. The fuel supply system pressurizes the fuel obtained from external oil sources into the high-pressure oil pipe and then into the cylinder of the engine. Therefore, the fuel parameters in each component of the fuel supply system influence each other. A mathematical model is established to describe the relationship between the fuel parameters of each part of the fuel supply system, and then the stability of the fuel supply system can be controlled by adjusting some parameters under given conditions.

The transfer of fuel in the fuel supply system can be analyzed finely by the differential element method [2], and the differential equations are established. When the differential equation is discretized, a set of recursive relations is obtained. After obtaining the initial state and related parameters, the fuel pressure parameters and fuel density parameters in each part of the fuel supply system at each time can be obtained recursively. Furthermore, the optimization model of fuel pressure control in high pressure fuel pipe can be established: the fuel supply duration of the one-way valve is used as the decision variable, and the difference between the fuel pressure at each time and the standard value is minimized as the optimization goal. The practical significance of each physical quantity is taken as the constraint condition.

In order to solve the optimization model in the case given in problem 1, it is necessary to find out the parameters. First of all, the analytical formula of elastic modulus and fuel pressure is fitted according to the experimental data; secondly, the relationship between fuel density and fuel pressure is obtained by solving differential equations. Then the pressure change law of high-pressure fuel pipe in the process of fuel injection is discussed and the characteristic function is introduced to describe the opening and closing law of valve; Similarly, the pressure change in the process of high-pressure fuel pump feeding into high-pressure fuel pipe is discussed. Finally, the model can be solved by substituting the parameters of each equipment into the optimization model.

Problem 2 changes the high-pressure fuel pump into an fuel pressing device composed of cam and plunger. Under this new condition, it is necessary to recalculate the parameters of the model. First of all, the relationship between the polar diameter and the polar angle of the cam can be obtained according to the edge curve of the cam; Secondly, the changing law of fuel volume and fuel density in the plunger cavity can be obtained by analyzing the motion law of the cam. Then, the opening and closing law of the injector can be obtained by analyzing the motion law of the needle valve at the nozzle. Accordingly, the objective function of the optimization model is kept unchanged, and the decision variable is changed to the angular velocity of the cam.

Question 3 adds a new fuel injection nozzle and an one-way pressure reducing valve on the basis of question 2 . Accordingly, when we analyze the fuel pressure of the high pressure fuel pipe in a very short time, we should consider the influence of the new nozzle and the one-way pressure reducing valve, which only needs to add two terms to the corresponding differential relation.

A study on the design of fuel pipelines. for example: According to the characteristics of the distributed structure of the accumulator volume, a novel optimisation idea to improve the steady-state performance of the high-pressure common-rail fuel injection system designed for a marine engine retrofitting is proposed [3]; Aiming at the problem that the dynamic characteristic of the sphere structure high speed on/off valve (HSV) is greatly affected by the supply pressure, a control algorithm for HSV that adaptive to supply pressure changes is proposed [4]; uses fuel in and out of the flow equation 、 the fluid flow equations, and other methods, the establishment of a suit-able, multi-objective optimization model of the system, by adopting the combination of quantitative analysis and qualitative analysis method to 
calibrate model parameters, in order to deal with high pressure tubing pressure control problem analysis [5]; experimental modal test results as well as finite element analysis of the fuel pipe due to the vibrational motion of an engine were reported [6]; uses infinitesimal method and multiple search algorithm to get recursive formula of density and pressure, and to obtain the pressure control strategy of the high-pressure fuel pipe hrough iteration [7].

\section{Model Building}

\subsection{Relationship Between Elastic Modulus E and Fuel Pressure $P$}

The relationship between elastic modulus $E$ and pressure $\mathrm{P}$ can be obtained by experimental method, as shown in Annex 3. Referring to the references, we can see that the elastic modulus of the gas spring is approximately linear with the pressure [9]. According to the corresponding values in Annex 3, the linear correlation coefficient of the data points is calculated, which is very close to 1 , so it can be approximately regarded as linear relationship. By using Matlab software for linear fitting, the approximate relationship $E-P$ is obtained as follows:

$$
E=8.9 P+1379.2
$$

Figure 3 shows the data scatter diagram and the fitting line comparison image in Annex 3, which shows that the fitting effect of the primary function is better.

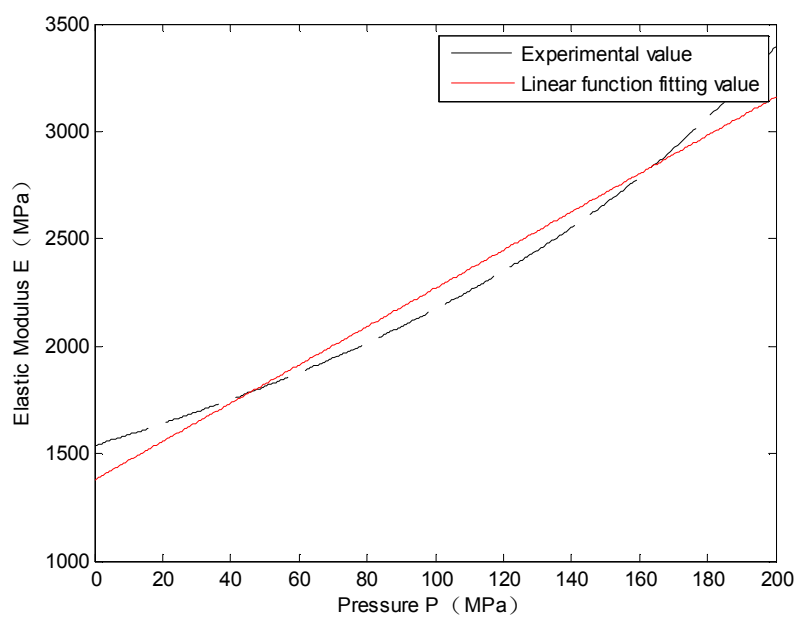

Figure 3. Scatter diagram and linear fitting comparison diagram of elastic modulus $E$ and fuel pressure $P$.

\subsection{Relationship Between Fuel Pressure P and Fuel Density $\rho$}

It can be seen from the question that the change of fuel pressure is proportional to the change of density, and the proportional coefficient is $\frac{E}{\rho}, E=E(P)$ is obtained by experimental method. Accordingly:

$$
\frac{\Delta P}{\Delta \rho}=\frac{E(P)}{\rho}
$$

Taking the minimal change process, the differential relation will be gotten,

$$
\frac{d P}{d \rho}=\frac{E(P)}{\rho}
$$

With the initial condition attached, When the pressure is 100 , the density of the fuel $\rho(100)=0.850$, the initial value problem of the following differential equation can be obtained,

$$
\left\{\begin{array}{l}
\frac{d P}{d \rho}=\frac{E(P)}{\rho} \\
\rho\left(P_{0}\right)=\rho_{0}
\end{array}\right.
$$

The relationship between fuel pressure $P$ and fuel density $\rho$ is obtained by solving the differential equation. In order to solve the problem conveniently, define $E=k P+b$, the constant $k=8.9, b=1379.2$.

By separating the variables from the differential equation, get

$$
\frac{d P}{k P+b}=\frac{d \rho}{\rho}
$$

Integral on both sides, get

$$
\frac{1}{k} \ln (k P+b)=\ln \rho+C_{1}
$$

Replace the initial condition $\rho=0.85$ and determine the integral constant $C_{1}=1.0307$. Therefore, the relationship $P$ and $\rho$ can be gotten:

$$
\rho=\rho(P)=e^{C_{1}}(k P+b)^{\frac{1}{k}}+C_{1} P=P(\rho)=\frac{1}{k}\left(\rho^{k} e^{k C_{1}}-b\right)
$$

It can be seen that $P$ is closely related to $\rho$, and if you give one of them, you can solve the other. Therefore, this paper only needs to find out the change law of the fuel density with time in the high-pressure fuel pipe.

\subsection{Mathematical Description of Fuel Supply Process of High Pressure Fuel Pump}

Assuming that the fuel supply starts at the moment $t$, the length of time for each opening of the one-way valve (the duration of a fuel supply) is recorded as $T_{A}$. At this time, the fuel pressure in the high-pressure fuel pipe $P=P(t)$ and the fuel pressure in the high-pressure fuel pump are recorded as $P_{A}=P_{A}(t)$. Therefore, the pressure difference between the high-pressure fuel pump and the high-pressure fuel pipe is:

$$
\triangle P=P_{A}(t)-P(t)
$$

Note that the length of the high-pressure fuel pipe is $L$, the inner diameter is $d$, and its volume can be obtained $V=\pi\left(\frac{d}{2}\right)^{2} L$. The area of the hole can be obtained according to the aperture of the oil supply nozzle $S_{A}=\pi\left(\frac{d_{A}}{2}\right)^{2}$.

Taking a short interval $[t, t+d t]$, fuel pressure $P(t)$ within high-pressure fuel pipe may be considered as unchanged during this period. According to the flow formula 
given by note 2 of the design, the flow rate $Q_{A}(t)$ of high-pressure fuel pump entering high-pressure fuel pipe is calculated within unit time (1 ms):

$$
Q_{A}(t)=C S_{A} \sqrt{\frac{2 \Delta P}{\rho_{A}}}
$$

Where, $C=0.85$ is the flow coefficient, $\rho_{A}$ is the density of the fuel on the side of the high-pressure fuel pump.

The volume of fuel flowing into the high-pressure fuel pipe in time $[t, t+d t]$ is: $Q_{A}(t) d t$, the mass of this part of the fuel is $\rho_{A} Q_{A}(t) d t$. Therefore, the fuel density $\rho(t+d t)$ in the high-pressure fuel pipe at $t+d t$ moment is,

$$
\rho(t+d t)=\frac{\rho(t) V+\rho_{A} Q_{A}(t) d t}{V}=\rho(t)+\frac{\rho_{A} Q_{A}(t) d t}{V}
$$

The change law of the fuel density in the high-pressure fuel pipe during the fuel supply process is described in this differential equation, and then according to the relationship $P=P(\rho)$, the change law $P(t)$ of the fuel pressure in the high-pressure fuel pipe can be gotten.

\subsection{Mathematical Description of Fuel Injection Process of High-Pressure Fuel Pump}

Assuming that fuel injection starts at a certain time $t$, the duration of each opening of injector $\mathrm{B}$ is recorded as $T_{B}$. At this time, the fuel pressure in the high-pressure fuel pipe is recorded as $P=P(t)$, the pressure in the cylinder of the external engine is $P_{G}$ (the engine starts to inhale new gas after the exhaust stroke ends, at this time, the cylinder has been emptied and its air pressure can be approximately constant $P_{G}=0$ ). Therefore, the pressure difference on both sides of the injector is,

$$
\Delta P=P(t)-P_{G}
$$

Taking a short time interval $[t, t+d t]$, the fuel pressure in the high-pressure fuel pipe during this period can be approximately regarded as unchanged. According to the flow formula given by note 2 of the design, the flow rate $Q_{B}(t)$ of high-pressure fuel pump entering high-pressure fuel pipe is calculated within unit time (1 ms):

$$
Q_{B}(t)=C S_{B} \sqrt{\frac{2 \Delta P}{\rho(t)}}
$$

Where, $C=0.85$ is the flow coefficient, $\rho(t)$ is the density of the fuel on the side of the high-pressure fuel pipe. The area of holes can be obtained according to nozzle $B$ diameter $d_{B}$. Therefore, the volume of fuel flowing into the high-pressure fuel pipe in time $[t, t+d t]$ is $Q_{B}(t) d t$, and the mass of this part of the fuel is $\rho_{B} Q_{B}(t) d t$. Therefore, the fuel density $\rho(t+d t)$ in the high-pressure fuel pipe at time $t+d t$ is,

$$
\rho(t+d t)=\frac{\rho(t) V-\rho(t) Q_{B}(t) d t}{V}=\rho(t)-\frac{\rho(t) Q_{B}(t) d t}{V}
$$

The changing law of the fuel density in the high-pressure fuel pipe during the fuel injection process is described in this differential equation, and then the changing law $P(t)$ of the fuel pressure in the high-pressure fuel pipe during the fuel injection process is gotten according to the relationship equation $P=P(\rho)$.

\subsection{The Change Law of the Pressure $P(t)$ in the High-Pressure Fuel Pipe During the Indirect Working Process of Fuel Feeding and Ejecting}

The working state of the high-pressure fuel pipe is divided into: fuel feeding, fuel injection, waiting. For the convenience of machining design, we assume that the fuel feeding operation is cyclically reciprocating, the machine starts to feed fuel for $T_{A} \mathrm{~ms}$ after it is turned on, then closes for $10 \mathrm{~ms}$ (the known conditions given in the design), and then feed fuel for $T_{A} \mathrm{~ms}, \cdots$, again and again. Similarly, the fuel injection operation starts injection $T_{B}$ ms after the machine is turned on, then shuts down for $100-T_{A} \mathrm{~ms}$ (injector works 10 times per second, so a working cycle is $100 \mathrm{~ms}$ ), and then injection $T_{B} \mathrm{~ms}, \cdots$, again and again. At a moment $\mathrm{t}$, the high-pressure fuel pipe may feed or inject fuel or may be in a waiting state. In order to find out the working state of the high-pressure fuel pipe at the moment, we define the characteristic function according to the periodicity of the working process $x_{\text {in }}(t)$ and $x_{\text {out }}(t)$ :

$$
\begin{gathered}
x_{\text {in }}(t)=\left\{\begin{array}{c}
1,0 \leq t \leq T_{A} \\
0, T_{A} \leq t \leq T_{A}+10 \\
x_{\text {in }}\left[t-\left(T_{A}+10\right)\right], t>T_{A}+10
\end{array}\right. \\
x_{\text {out }}(t)=\left\{\begin{array}{c}
1,0 \leq t \leq T_{B} \\
0, T_{B} \leq t \leq 100 \\
x_{\text {out }}(t-100), t>100
\end{array}\right.
\end{gathered}
$$

With the help of the characteristic function to judge whether the fuel is entering and exiting, we can easily consider all kinds of conditions in the working process. Next, the law of change is established by using the following steps. At any time $t$, the fuel pressure in the high-pressure fuel pipe $P=P(t)$, the pressure in the cylinder of the external engine $P_{G}=0$ and the fuel pressure in the high-pressure pump is $P_{A}$. Taking a very short time interval $[t, t+d t]$, during which the fuel pressure $P(t)$ in the high-pressure fuel pipe can be approximately regarded as unchanged. According to the flow formula, the fuel supply flow rate $Q_{A}(t)$ and injection flow rate $Q_{B}(t)$ in unit time $(1 \mathrm{~ms})$ can be calculated. According to the characteristic function to judge whether the process is fuel feeding and injection, it is obtained that the volume of fuel flowing into the high pressure fuel pipe in time $[t, t+d t]$ is $x_{i n}(t) Q_{A}(t) d t$, the mass of this part of fuel is: $x_{i n}(t) \rho_{A}(t) Q_{A}(t) d t$, the volume of fuel flowing out of the high-pressure fuel pipe in time is: $x_{\text {out }}(t) Q_{B}(t) d t$, the mass of this part of fuel is $x_{\text {out }}(t) \rho(t) Q_{B}(t) d t$. Therefore, the fuel density in the high-pressure fuel pipe becomes:

$$
=\frac{\rho(t) V+x_{\text {in }}(t) \rho_{A}(t) Q_{A}(t) d t-x_{\text {out }}(t) \rho(t) Q_{B}(t) d t}{V}
$$




$$
=\rho(t)+\frac{x_{\text {in }}(t) \rho_{A}(t) Q_{A}(t) d t-x_{\text {out }}(t) \rho(t) Q_{B}(t) d t}{V}
$$

The differential equation can also be rewritten as:

$$
\frac{d \rho}{d t}=\frac{x_{\text {in }}(t) \rho_{A}(t) Q_{A}(t)}{V}-\frac{x_{\text {out }}(t) \rho(t) Q_{B}(t)}{V}
$$

According to the initial state of the high-pressure fuel pipe, it is easy to get its initial condition: $\rho(0)=\rho_{0}$.

The changing law of the fuel density $\rho(t)$ in the high-pressure fuel pipe at any time can be described in this differential equation (the working state of the fuel supply system may be fuel injection, fuel feeding or waiting). The fuel density of the high-pressure fuel pipe $\rho(t)$ at any time $\mathrm{t}$ will be gotten by solving the differential equation. According to the relational formula $P=P(\rho)$, the change rule of fuel pressure in the high-pressure fuel pipe during fuel injection $P(t)$ is obtained.

\subsection{The Optimal Model of Pressure $P(t)$ Control in High-Pressure Oil Pipe}

In order to check whether the engine fuel supply is stable or not, a long enough observation time $T$ can be selected, and the recursive relation is used to iteratively calculate the pressure $P(t)$ in the high pressure fuel pipe at each time in $[0, T]$. The deviation of $P(t)$ and Rating $P_{e}$ measures the stability of the engine fuel supply system. Therefore, we construct the objective function as follows:

$$
\operatorname{Min} Z=\int_{0}^{T}\left|P(t)-P_{e}\right| d t
$$

Where, the decision variable is the length of time for each opening of the one-way valve $T_{A}$.

Considering the practical significance, the constraints are increased: $0<T_{A}<T$.

\subsection{Discretization Algorithm of $P(t)$ Control Optimization Model}

Although the differential equation of $P(t)$ can determine $P(t)$, it is difficult to solve it. Therefore, the discretization method can be used for numerical solution. The specific algorithm steps are as follows:

Step 1 Initialize the preparation work. Determining the initial state of the fuel supply system, which includes the initial state of the high pressure fuel pipe, that is fuel pressure $P(0)$, fuel density $\rho(0)$, and the initial state of the high pressure fuel pump, that is fuel pressure $P_{A}(0)$ and fuel density $\rho_{A}(0)$. The initial state of the engine side is fuel pressure $P_{G}$ and fuel density $\rho_{G}$ (it has been assumed that the fuel pressure and fuel density on the engine side are always constant). Choosing Choose an appropriate time interval $\mathrm{dt}$ (for example, $0.01 \mathrm{~ms}$ ) and a long enough observation period $[0, T]$.

Step 2 The decision variable $\omega$ is cyclically changed. After setting cycle change decision variables, the fuel supply judgment characteristic function $x_{i n}(t)$ and the fuel injection judgment characteristic function $x_{\text {out }}(t)$ can be determined. Discretizing the differential equation into difference equation:

$$
\rho(t+d t)=\rho(t)+\frac{x_{\text {in }}(t) \rho_{A}(t) Q_{A}(t) d t-x_{o u t}(t) \rho(t) Q_{B}(t) d t}{V}
$$

On the right side of the equation are the known quantities of the moment $\mathrm{t}$, and the physical quantities $\rho(t+d t), P(t+d t)$ of the next moment $t+d t$ can be obtained by using the recursive iteration. If you continue to iterate, you will get the pressure value of the high-pressure fuel pipe at each moment.

$$
P(0), P(0+d t), P(0+2 d t), \cdots, P(T)
$$

The sum of the deviations between the pressure values and the ratings at these moments is calculated to equivalent replace the objective function in the optimization model.

$$
S\left(T_{A}\right)=\sum_{i=1}\left|P(0+i d t)-P_{e}\right| d t
$$

\section{Solution of the Model}

\subsection{Solution of the Model in the Case of Question 1}

The optimization model of $P(t)$ has been established and the discretization solution method has been given. Combined with these specific data that given in Question 1, each parameter of the model can be calculated, then specific solution model can be obtained. Although some specific relationships such as $E=8.9 P+1379.2$ have been calculated above, these relationships are listed briefly here again for the sake of clarity.

\subsubsection{Relationship Between Elastic Modulus E and Fuel Pressure $P$}

The formula of $E-P$ has been obtained by using the method of data fitting:

$$
E=k P+b
$$

Where, constant $k=8.9, b=1379.2$.

\subsubsection{Relationship Between Elastic Modulus E and Fuel Density $\rho$}

In chapter 2 , the relation of $P-\rho$ has been obtained by solving differential equations:

$$
\begin{aligned}
& \rho=\rho(P)=e^{-C_{1}}\left(k P+b^{\frac{1}{k}}\right) \\
& P=P(\rho)=\frac{1}{k}\left(\rho^{k} e^{k C_{1}}-b\right)
\end{aligned}
$$

Where, constan $k=8.9, b=1379.2, C_{1}=\frac{1}{k} \ln (100 k+$ b) $-\ln 0.85$

\subsubsection{The Flow Function $Q_{A}(t)$ and the Characteristic Function $\boldsymbol{x}_{\text {in }}(\boldsymbol{t})$}

The flow function $Q_{A}(t)$ of the fuel supply process is:

$$
Q_{A}(t)=C S_{A} \sqrt{\frac{2 \Delta P}{\rho_{A}}}=C S_{A} \sqrt{\frac{2\left[P_{A}-P(t)\right]}{\rho_{A}}}
$$

Where, the flow coefficient $C=0.85$, and the area of the hole $S_{A}=\pi\left(\frac{d_{A}}{2}\right)^{2}=1.5394$. The density of fuel $\rho_{A}$ on the side of the high pressure oil pump is a fixed value, which can be 
calculated from the formula $\rho_{A}=\rho\left(P_{A}\right)=e_{C 1}\left(k P+b^{\frac{1}{k}}\right)=$ 0.8704 .

Assuming that the fuel supply $\mathrm{A}$ open for $T_{A}$ each time, and then closed for $10 \mathrm{~ms}$, and then cycled periodically, thus the characteristic function $x_{i n}(t)$ for fuel supply judgment is as follows:

$$
x_{i n}(t)=\left\{\begin{array}{c}
1,0 \leq t \leq T_{A} \\
0, T_{A} \leq t \leq T_{A}+10 \\
x_{i n}\left[t-\left(T_{A}+10\right)\right], t>T_{A}+10
\end{array}\right.
$$

\subsubsection{The Flow Function of the Fuel Injection Process \\ $Q_{B}(t)$ and the Characteristic Function $x_{\text {out }}(t)$}

While the diameter of hole B is not given in Question 1, the fuel injection rate in a cycle when the injector working normally, that is, the flow rate $Q_{B}(t)$, is given directly by the image which is shown in Figure 4.

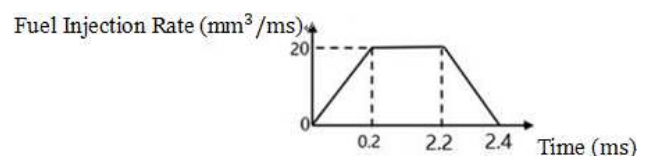

Figure 4. Schematic diagram of the velocity of injector $B$

From the Figure 4, it can be seen that the injection rate is stable at 20 under the standard state where the pressure in the high pressure fuel pipe $P(t)=100$.

$$
Q_{B}(t)=C S_{B} \sqrt{\frac{2 \Delta P}{\rho(t)}}=C S_{B} \sqrt{\frac{2\left[P_{A}-P_{G}\right]}{P(t)}}
$$

It can obtain $S_{B}=16.6378$, what's more, an implicit condition that can inferred that the diameter of the hole $\mathrm{B}$ in question $1 d_{B}=4.6026$.

On the other hand, the fuel injection rate rises linearly from 0 to $0.2 \mathrm{~ms}$, and then stabilizes at 20 . It means that the opening of fuel injector B requiring a process, which takes $0.2 \mathrm{~ms}$ to complete the valve from fully closed to fully open. At the same, it can be regarded as the closing process of the valve within 2.2 to $2.4 \mathrm{~ms}$. It can be inferred that the switch of the valve is a device similar to a spring, and the buffer time of the switch is related to the mechanical properties of itself. For the sake of simplification, it is assumed that the opening and closing buffer time of valve $\mathrm{B}$ is constant at 0.2 $\mathrm{ms}$, which is independent of fuel pressure.

In order to describe the opening and closing process of the valve in detail, we set the characteristic function $x_{\text {out }}(t)$ describing whether the nozzle is open to a fuzzy value, which is no longer limited to number between 0 and 1 can be taken to express the state of partial opening of the valve (e.g. 0.5 means that the valve is half open). The valve B gradually open Within 0-0.2 $\mathrm{ms}$ and the corresponding feature function increases linearly from 0 to 1 (for the sake of simplification, the feature function is regarded as a linear function about time). The valve $B$ remains open and the characteristic function remains at 1 within $0.2-2.2 \mathrm{~ms}$. The valve $B$ gradually closes and the corresponding characteristic function decreases linearly from 1 to 0 within 2.2-2.4 milliseconds. Therefore, the periodic characteristic function $x_{\text {out }}(t)$ can be obtained as follows:

$$
x_{\text {out }}(t)=\left\{\begin{array}{c}
5 t, 0 \leq t<0.2 \\
1,0.2 \leq t<T_{B}-0.2 \\
1-5(t-2.2), T_{B}-0.2 \leq t<T_{B} \\
0, T_{B} \leq t \leq 100 \\
x_{\text {out }}(t-100), t>100
\end{array}\right.
$$

Where, it is known that the length of time for the valve $B$ to be opened at one time $T_{B}=2.4$.

When the air pressure of the high-pressure fuel pipe $P(t)$ is not at the standard value of 100 , the speed of the fuel injection nozzle $Q_{B}(t)$ is still determined by the flow formula:

$$
Q_{B}(t)=C S_{B} \sqrt{\frac{2 \Delta P}{\rho(t)}}=C S_{B} \sqrt{\frac{2\left[P(t)-P_{G}\right]}{P(t)}}
$$

Where, flow coefficient $C=0.85$, the area of the hole $S_{B}=16.6378$, and the pressure on the engine side is approximately zero $P_{G}=0$. By substituting these parameters into the flow formula, you can get

$$
Q_{B}(t)=C S_{B} \sqrt{\frac{2 \Delta P}{\rho(t)}}=C S_{B} \sqrt{\frac{2\left[P(t)-P_{G}\right]}{P(t)}}=20
$$

It can be seen that at the injection nozzle $B$, the flow velocity $Q_{B}(t)$ does not change with the change of pressure, and is always $Q_{B}(t) \equiv 20$.

\subsubsection{Variation Law of Fuel Density $\rho(t)$ and Pressure $P(t)$ in High Pressure Fuel Pipe}

It has been concluded in $\S 4$ that the satisfied differential equation $P(t)$ is:

$$
\frac{d \rho}{d t}=\frac{x_{\text {in }}(t) \rho_{A}(t) Q_{A}(t)}{V}-\frac{x_{\text {out }}(t) \rho(t) Q_{B}(t)}{V}
$$

Discretizing this differential equation, and the satisfied recurrence relation is

$$
\rho(t+d t)=\rho(t)+\frac{x_{\text {in }}(t) \rho_{A}(t) Q_{A}(t) d t-x_{o u t}(t) \rho(t) Q_{B}(t) d t}{V}
$$

Where, the volume of high-pressure fuel pipe $V=39270$.

The initial conditions: $\rho(0)=0.85$.

\subsubsection{Optimized $T_{A}$ to Stabilize the Fuel Pressure in the High-Pressure Fuel Pipe at 100}

The optimization goal is:

$$
\operatorname{Min} S\left(T_{A}\right)=\sum_{i=1}\left|P(0+i d t)-P_{e}\right| d t
$$

Constraint condition: $0<T_{A}<T$, where, the observation period $\mathrm{T}$ can be selected according to the need.

\subsubsection{Discretization Algorithm for Solving $P(t)$ Control Optimization Model}

Although the differential equation about $P(t)$ can be determined, it is difficult to solve. Therefore, the discretization method is used for numerical solution in this paper. The specific steps of the algorithm are as follows:

Step 1 Initialization preparations. Recording the initial state of the high-pressure fuel pipe that is fuel pressure 
$P(0)=100$, fuel density $\rho(0)=0.85$, the initial state of the high-pressure fuel pump that is fuel pressure $P_{A}(0)=160$, fuel density $\rho_{A}(0)=0.8704$, the initial state of the engine side is fuel pressure $P_{G}=0$, fuel density $\rho_{G}=0$. Choosing an appropriate interval $=0.01$, Observation period $[0, T]$, $T=10 \mathrm{~s}=10000 \mathrm{~ms}$ is selected.

Step 2 Cyclic change of decision variables $T_{A} \in(0, T)$.

After determining $T_{A}$, the characteristic function of fuel supply $x_{i n}(t)$ can be determined.

Then solving $Q_{B}(t)$ (it is constant 20), $Q_{A}(t)$.

Next, the iterative recursive relationship:

$$
\rho(t+d t)=\rho(t)+\frac{x_{\text {in }}(t) \rho_{A}(t) Q_{A}(t) d t-x_{\text {out }}(t) \rho(t) Q_{B}(t) d t}{V}
$$

The right side of the equation are the known quantities of the moment $t$, and the physical quantities of the next moment $t+d t$ can be obtained by iterating one step with the recursive formula $\rho(t+d t), P(t+d t)$.

Continue to iterate, the pressure value of the high-pressure fuel pipe at each time will be gotten.

$$
P(0), P(0+d t), P(0+2 d t), \cdots P(T)
$$

In particular, the pressure value (2000) at 2 seconds, the pressure value at 5 seconds (5000), and the pressure value at 10 seconds (10000) are stored for use.
Calculating the sum of the deviations between the pressure values and the ratings at these moments:

$$
S\left(T_{A}\right)=\sum_{i=1}\left|P(0+i d t)-P_{e}\right| d t
$$

Step 3 Changing the decision variable $T_{A} \in(0, T)$ to find out the minimum value of the deviation $S$, which corresponds $T_{A}^{*}$ to the optimal fuel supply time, that is,

$$
S\left(T_{A}^{*}\right)=\underset{T_{A} \in(0, T)}{\operatorname{Min}} S\left(T_{A}\right)
$$

The program is written with Mathematica software to solve the optimization model. In the process of solving the model, some of the values searched are listed in Table 1.

In order to minimize the error, the optimal fuel supply time is obtained by searching $T_{A}^{*}=0.29 \mathrm{~ms}$.

If the fuel pressure of the high pressure fuel pipe is increased to 150 and stabilized at 150 after 2 seconds, it is only necessary to change the objective function in the optimization model: minimize the error between the pressure and 150 after 2 seconds, and increase the constraint condition $P(2000) \geq 150$. By computer search, the optimal fuel supply time $T_{A}^{*}=0.86 \mathrm{~ms}$ is obtained. Some of the search results in the solution process are shown in Table 2.

Table 1. The error between the pressure in the high-pressure fuel pipe and the standard pressure 100 under the condition of different fuel supply time.

\begin{tabular}{lllll}
\hline \multirow{2}{*}{ fuel supply time $\boldsymbol{T}_{\boldsymbol{A}}$} & $\begin{array}{l}\text { The pressure at 2s } \\
\boldsymbol{P ( 2 0 0 0 )}\end{array}$ & $\begin{array}{l}\text { The pressure at 5s } \\
\boldsymbol{P ( 5 0 0 0 )}\end{array}$ & $\begin{array}{l}\text { The pressure at 10s } \\
\boldsymbol{P 1 0 0 0 0 )}\end{array}$ & $\begin{array}{l}\text { Error between pressure and standard pressure } \\
\text { within 10s }\end{array}$ \\
\hline 1 & 153.8543 & 155.4610 & 155.6329 & 502590 \\
0.5 & 126.1389 & 138.1154 & 140.0343 & 314590 \\
0.3 & 102.3237 & 104.0432 & 105.1219 & 24946.3889 \\
0.25 & 95.1959 & 90.3301 & 86.4599 & 98609.0042 \\
0.2 & 87.6359 & 74.8517 & 62.2865 & 242831.4175 \\
\hline
\end{tabular}

If the fuel pressure of the high pressure fuel pipe is increased to 150 and stabilized at 150 after 5 seconds, it is only necessary to change the objective function in the optimization model to: minimize the error between $P(t)$ after 5 seconds and 150 , and increase the constraint condition $P(5000) \geq 150$. The optimal fuel supply time $T_{A}^{*}=0.75 \mathrm{~ms}$ is obtained by cyclic search. Some of the search results in the solution process are listed in Table 2 .

Table 2. The error between the pressure in the high-pressure fuel pipe and the standard pressure 150 under the condition of different fuel supply time.

\begin{tabular}{lllll}
\hline fuel supply time & $\begin{array}{l}\text { The pressure at 2s } \\
\boldsymbol{P}(\mathbf{2 0 0 0})\end{array}$ & $\begin{array}{l}\text { The pressure at 5s } \\
\boldsymbol{P ( 5 0 0 0 )}\end{array}$ & $\begin{array}{l}\text { The pressure at 10s } \\
\boldsymbol{P 1 0 0 0 0 )}\end{array}$ & $\begin{array}{l}\text { Cumulative error between pressure and standard } \\
\text { pressure 150 in 5-10 s }\end{array}$ \\
\hline $\boldsymbol{T}_{\boldsymbol{A}}$ & 151.3312 & 154.1801 & 154.2605 & 13970.0001 \\
0.9 & 147.6559 & 152.3680 & 152.4100 & 5682.6103 \\
0.7 & 142.0512 & 149.9404 & 150.0179 & 7224.3693 \\
0.6 & 135.0614 & 145.5089 & 146.2694 & 26674.3516 \\
0.5 & 126.1388 & 138.1154 & 140.0343 & 59692.77794 \\
\hline
\end{tabular}

If the fuel pressure of the high pressure fuel pipe is increased to 150 and stabilized at 150 after 10 seconds, it is only necessary to change the objective function in the optimization model: minimize the error between $P(t)$ after 10 seconds and 150, and increase the constraint condition $P(10000) \geq 150$. Because the longer the fuel supply, the faster the pressure increases, we only need to increase $T_{A}$ gradually to make just $P(10000) \geq 150$, which the smallest one meets the requirements of the model. Programming search to get the optimal fuel supply time $T_{A}=0.7 \mathrm{~ms}$. Some of the search results in the solution process are shown in Table 3.

Table 3. The pressure value of the 10th second in the high-pressure tubing under the condition of different fuel supply time.

\begin{tabular}{llll}
\hline Fuel supply time $\boldsymbol{T}_{\boldsymbol{A}}$ & $\mathbf{0 . 9}$ & $\mathbf{0 . 8 5}$ & \multicolumn{1}{c}{$\mathbf{0 . 8}$} \\
\hline The pressure in the high-pressure fuel pipe at the 10s $P(10000)$ & 154.2605 & 153.4107 & 152.4100 \\
Fuel supply time $T_{A}$ & 0.7 & 0.65 & 0.6 \\
The pressure in the high-pressure fuel pipe at the 10s $P(10000)$ & 150.0179 & 148.2163 & 146.2694 \\
\hline
\end{tabular}




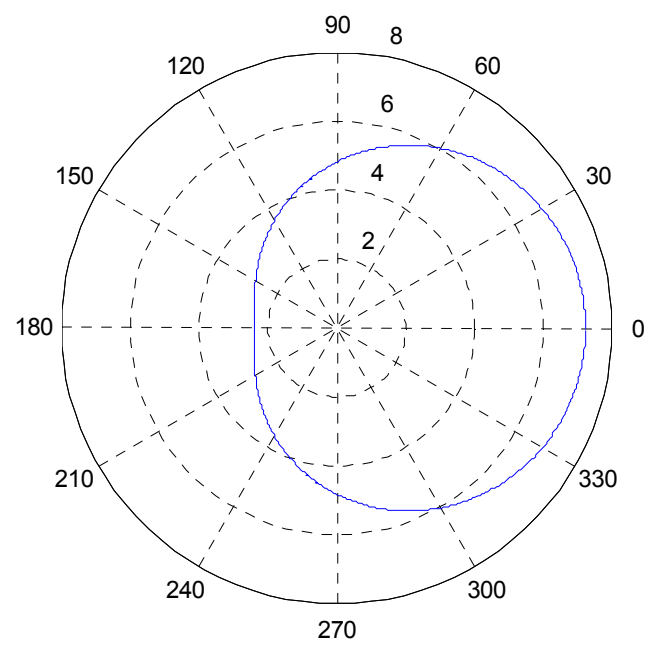

Figure 5. Cam edge curve in polar coordinate system.

\subsection{Solution of the Model in the Case of Problem 2}

The conditions given in question (2) are more complex and more similar to the actual fuel supply system. Among them, high-pressure fuel pump is no longer an ideal stable fuel source, and its fuel pressure $T_{A}=T_{A}(t)$ changes periodically with the rotation of cam; The fuel injection rule of the fuel injector B is determined by the movement of the needle valve, so the fuel injection characteristic function $x_{\text {out }}(t)$ also changes accordingly. As in problem 1, we find out the parameters of the model under this new condition, and then substitute the optimization model established in $\S 2$ to solve it.

\subsubsection{Determination of the Cam Edge Curve}

Annex 1 has measured the edge curve of the cam, read in these data, and made the cam curve diagram as shown in Figure 5.

Because the expression of the cam curve is more complicated [10], This article regards the polar angle-polar diameter corresponding data given in Annex 1 as the discrete form of the cam curve equation, When the polar angle is give, the polar diameter can be found by periodically converting the angle to 0-6.27 and then using a look-up table. A part of the corresponding data of polar angle and polar diameter in Annex 1 is listed in table 4:

Table 4. Polar angle-polar diameter of cam edge curve.

\begin{tabular}{|c|c|c|c|c|c|c|c|c|c|c|}
\hline Polar angle (rad) & $\mathbf{0}$ & 0.1 & 0.2 & 0.3 & 0.4 & 0.5 & 0.6 & 0.7 & 0.8 & 0.9 \\
\hline polar diameter $(\mathrm{mm})$ & 7.239 & 7.2389 & 7.2385 & 7.2379 & 7.2371 & 7.236 & 7.2347 & 7.2331 & 7.2313 & 7.2292 \\
\hline
\end{tabular}

\subsubsection{Variation Law of Fuel Volume $V_{z h u}(t)$ in Plunger Cavity During Cam Rotation}

In the polar coordinate system, consider the movement law of the cam and the plunger contact point $J(\theta(t)), r(\theta(t))$. The cam rotates at an angular velocity $\omega$, the initial position of point $J$ is $\theta_{0}=\frac{\pi}{2}$, then at time $t$

$$
\begin{aligned}
& \theta(t)=\theta_{0}+\omega \\
& r(t)=r(\theta(t))
\end{aligned}
$$

According to Annex 1, on the edge curve of the cam, the minimum pole diameter $r_{\text {min }}=2.4130$ and the maximum pole diameter $r_{\max }=7.2390$. The difference between the two is the maximum stroke of the piston $h_{z h u}=r_{\max }-r_{\min }=$ 4.8260 , the minimum volume in the cavity during the movement of the piston is $V_{\min }=20$. The known inner diameter of the plunger cavity $d_{z h u}=5$, and its cross-sectional area $\pi\left(\frac{d_{z h u}}{2}\right)^{2}=19.6350$, the maximum volume of fuel in the plunger cavity is:

$$
V_{\text {max }}=V_{\min }+\pi\left(\frac{d_{z h u}}{2}\right)^{2} h_{z h u}
$$

The volume of fuel in the plunger cavity at time $t$ can be calculated as:

$$
V_{z h u}=V_{\max }-\pi\left(\frac{d_{z h u}}{2}\right)^{2}\left(r(t)-r_{\min }\right)
$$

The change rule of the fuel volume in the plunger cavity during the cam rotation is obtained, where the polar diameter of the initial point $J$ is $r=r\left(\theta_{0}\right)=r \frac{\pi}{2}=4.8279$, and the corresponding fuel volume $V_{z h u}(0)=V_{\text {max }}-\pi\left(\frac{d_{z h u}}{2}\right)^{2}\left(r(t)-r_{\text {min }}\right)=67.3418$.

\subsubsection{Variation Law of Fuel Density $\rho_{A}(t)$ and Pressure $P_{A}(t)$ in the Plunger Cavity}

Taking a very short time course $[t, t+d t]$ and perform a small amount analysis. In this process, the height of the plunger rise is $d r=r(t+d t)-r(t)$; The volume of fuel in the plunger cavity also becomes $V_{z h u}(t+d t)$. The expression for this volume has been given in Section 3.1,

If the pressure in the plunger cavity is greater than the pressure in the high-pressure fuel pipe, the one-way valve A opens and the fuel supply process starts, otherwise no fuel is supplied. Therefore, the characteristic function for judging fuel supply is modified as:

$$
x_{i n}(t)\left\{\begin{array}{l}
1, P_{A}(t)>P(t) \\
0, P_{A}(t) \leq P(t)
\end{array}\right.
$$

or

$$
x_{i n}(t)\left\{\begin{array}{l}
1, \rho_{A}(t)>\rho(t) \\
0, \rho_{A}(t) \leq \rho(t)
\end{array}\right.
$$

If the period $[t, t+d t]$ belongs to the fuel supply phase, the fuel supply rate $Q_{A}(t)$ is:

$$
Q_{A}(t)=C S_{A} \sqrt{\frac{2 \Delta P}{\rho_{A}}}=C S_{A} \sqrt{\frac{2\left[P_{A}-P(t)\right]}{\rho_{A}}}
$$


The fuel supply volume during this period is $Q_{A}(t) d t$, and its mass is $\rho_{A}(t) Q_{A}(t) d t$. Considering the compression process and fuel supply process comprehensively, the fuel density in the plunger cavity at $t+d t$ can be determined by the recursive relationship as follows:

$$
\rho_{A}(t+d t)=\frac{\rho_{A}(t) V_{z h u}(t) d t-x_{i n}(t) \rho_{A}(t) Q_{A}(t) d t}{V_{z h u}(t+d t)}
$$

Every time the plunger moves to the bottom dead center, the external low-pressure fuel (constant pressure 0.5) will fill the plunger cavity, so when the smallest point of the pole diameter on the cam turns to the contact point, $P_{A}(t)$ will automatically reset to $P_{A}(t)=0.5$, the pressure is converted into the corresponding density, and $\rho_{A}(t)$ will be reset to $\rho_{A}(t)=8040$. At this moment, the polar angle of point $J$ is:

$$
\theta(t)=\pi, \pi+2 \pi, \pi+4 \pi, \cdots, \pi+2 n \pi n \in N
$$

In this way, we get the variation law of fuel density (pressure) in the plunger cavity.

Next, determine the initial state of the fuel in the plunger cavity. Since the fuel in the plunger cavity must be reset every cycle, the initial pressure and density values only affect the data in a very short time (less than one cycle). Based on this, we simply set the initial value Set as $P_{A}(0)=0.5, \rho_{A}(0)=0.80458$.

\subsubsection{Characteristic Function $x_{\text {out }}(t)$ for Judging Whether to Inject Fuel}

Annex 2 gives the needle valve lift data in a period of 100 milliseconds, based on which Figure 6 can be made.

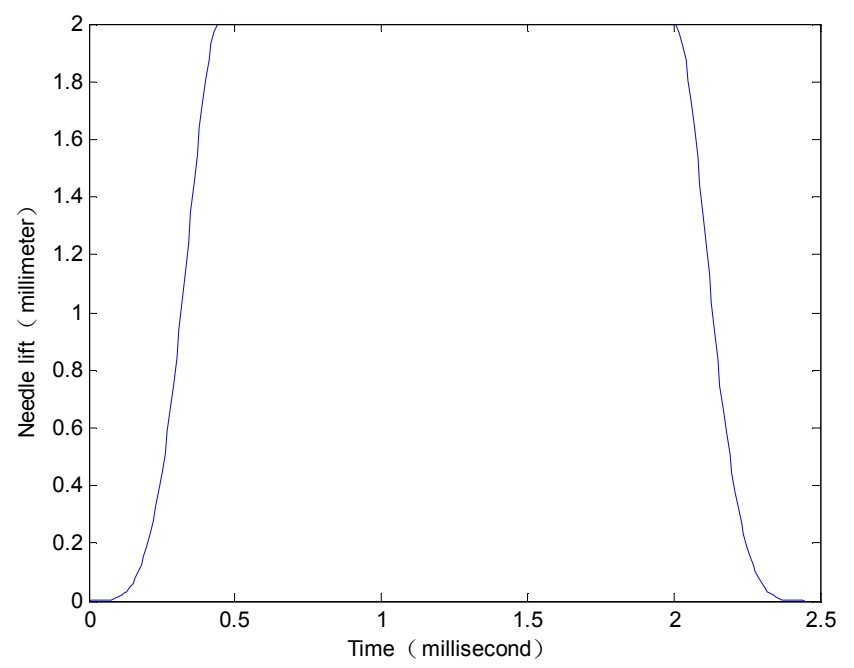

Figure 6. The lift of the needle valve changes with time during the operation of the fuel injector.

From Figure 6, it can be seen that from 0 to 0.45 milliseconds, the needle valve gradually rises and the fuel injector B gradually opens; then the needle valve is stable within 0.45-2 milliseconds (the lift remains at $2 \mathrm{~mm}$ ), the fuel injector B is fully open; within 2-2.45 milliseconds, the needle valve gradually drops, and the fuel injector $B$ gradually closes. The opening and closing buffer time of the fuel injector B is about 0.45 milliseconds.

For the sake of simplicity, it is assumed that the opening and closing buffer process of the fuel injector B is regarded as a linear function. So, similar to question 1, write the characteristic function $x_{\text {out }}(t)$ describing the opening and closing law of valve $\mathrm{B}$ as follows:

$$
x_{\text {out }}(t)=\left\{\begin{array}{c}
\frac{1}{0.45}, 0 \leq t<0.45 \\
1,0.45 \leq t<2 \\
1-\frac{1}{0.45}(t-2), 2 \leq t<2.45 \\
0, T_{B} \leq t \leq 100 \\
x_{\text {out }}(t-100), t>100
\end{array}\right.
$$

\subsubsection{Flow Function $Q_{B}(t)$ During Fuel Injection}

Question 2 gives the diameter of hole $\mathrm{B} d_{B}=1.4$, and the area of hole $\mathrm{B}$ is $\pi\left(\frac{d_{B}}{2}\right)^{2}=1.5394$. It is known that the flow coefficient $C=0.85$, and it has been assumed that the pressure on the engine side is approximately zero, ie $P_{G}=0$. Substitute the flow formula to get the flow during the fuel injection process

$$
Q_{B}(t)=C A_{B} \sqrt{\frac{2 \Delta P}{\rho(t)}}=C A_{B} \sqrt{\frac{2\left[P_{A}-P_{G}\right]}{P(t)}}=1.8505
$$

The density in the high-pressure fuel pipe is still updated by the recurrence equation given in Chapter 2 :

$$
\rho(t+d t)=\rho(t)+\frac{x_{\text {in }}(t) \rho_{A}(t) Q_{A}(t) d t-x_{o u t}(t) \rho(t) Q_{B}(t) d t}{V}
$$

\subsubsection{The Recursive Method of Solving Without the \\ Conversion Function of Pressure and Density}

We start from the differential relationship $\frac{d P}{d \rho}=\frac{E(P)}{\rho}$, discretize it, and get the recurrence relationship

$$
\frac{P(t+d t)-P(t)}{\rho(t+d t)-\rho(t)}=\frac{E(P(t))}{\rho(t)}
$$

Where, the parameters $P(t)$ and $\rho(t)$ at time t can be regarded as known in the recursive process; and $E(P(t))$ can be obtained by checking the table in Annex 3; and $\rho(t+d t)$ can be The previous recurrence relation is obtained, and therefore:

$$
P(t+d t)=P(t)+\frac{E(P(t))}{\rho(t)}[\rho(t+d t)-\rho(t)]
$$

If the exact formula of $E(P)$ can be obtained but the differential equation $\frac{d P}{d \rho}=\frac{E(P)}{\rho}$ cannot be solved, the conversion formula of $P(t)$ and $\rho(t)$ cannot be obtained. In this case, the recursive method can be used to obtain the fuel parameter $\rho(t+d t), P(t+d t)$ at time $t+d t$ from the fuel parameters $P(t), \rho(t)$ and $E(P(t))$ at time $\mathrm{t}$.

In particular, combined with the initial conditions: when $P$ $=100, \rho=0.850$, the corresponding density under each pressure value can be calculated. Such as by

$$
\frac{100-99.5}{0.85-\rho(99.5)}=\frac{2171.4}{0.85}
$$

It is easy to figure out the corresponding density value 
$\rho(99.5)=0.8498$ when the pressure is 99.5 . In the same way, starting from the initial conditions and iterating forward and backward, a series of pressure and density corresponding value tables can be obtained. Part of the results are listed in Table 5, and the entire data list is shown in supporting materials.

Table 5. Starting from the initial conditions, iteratively calculates the partial corresponding value table of pressure and density forward and backward.

\begin{tabular}{llllllllll}
\hline Pressure $\boldsymbol{P}$ & $\mathbf{0}$ & $\mathbf{0 . 5}$ & $\mathbf{1}$ & $\mathbf{1 . 5}$ & $\mathbf{2}$ & $\mathbf{2 . 5}$ & $\mathbf{3}$ & $\mathbf{3 . 5}$ \\
\hline Density $\rho$ & 0.8043 & 0.8046 & 0.8048 & 0.8051 & 0.8053 & 0.8056 & 0.8058 & 0.8061 & 0.8063 \\
Pressure $P$ & 5 & 5.5 & 6 & 6.5 & 7 & 7.5 & 8 & 8.5 \\
Density $\rho$ & 0.8069 & 0.8071 & 0.8074 & 0.8076 & 0.8079 & 0.8082 & 0.8084 & 0.8087 & 0.8089 \\
\hline
\end{tabular}

\subsubsection{Optimization to Stabilize the Fuel Pressure in the}

\section{High-Pressure Fuel Pipe at 100}

The optimization goal is: $\operatorname{Min} S(\omega)=\sum_{i=1} \mid P(0+i d t)-$ $P_{e} \mid d t$

According to the actual meaning, the constraint condition is given: $\omega>0$, where the observation period can be selected artificially.

Through Baidu search, the general speed of the cam is about $100 \mathrm{rpm}$., the speed higher than $200 \mathrm{rpm}$ is called high speed, and the speed lower than $100 \mathrm{rpm}$ is called low speed. Therefore, the speed range is about $0-1000 \mathrm{rpm}$, which is $0-0.10472$ radians $/ \mathrm{ms}$.

\subsubsection{Discretization Algorithm of $P(t)$ Control Optimization Model}

Step 1 Initialization preparations. Record the initial state of the high-pressure fuel pipe that is fuel pressure $P(0)=100$, fuel density $\rho(0)=0.850$, the initial state of the high-pressure fuel pump is fuel pressure $P_{A}(0)=0.5$, fuel density $\rho_{A}(0)=0.8040$, engine The initial state of the side is fuel pressure $P_{G}=0$, fuel density $\rho_{G}=0$. Choose an appropriate time step $d t=0.01$, observation period $[0, T]$, $T=10 \mathrm{~s}=10000 \mathrm{~ms}$ can be selected.

Step 2 The decision variable $\omega$ is cyclically changed. After $\omega$ is determined, the fuel supply characteristic function $x_{i n}(t)$ can be determined; then $Q_{B}(t)$ (constant 1.8505), $Q_{A}(t)$, according to the position of the cam at the current moment, determine whether to reset $\rho_{A}, P_{A}$.

Next, the iterative recursion relationship:

$$
\begin{gathered}
\rho_{A}(t+d t)=\frac{\rho_{A}(t) V_{z h u}(t) d t-x_{\text {in }}(t) \rho_{A}(t) Q_{A}(t) d t}{V_{z h u}(t+d t)} \\
\rho(t+d t)=\rho(t)+\frac{x_{\text {in }}(t) \rho_{A}(t) Q_{A}(t) d t-x_{o u t}(t) \rho(t) Q_{B}(t) d t}{V}
\end{gathered}
$$

The right side of the equation is the known quantity of the time. Using this recursion to iterate one step, you can get the physical quantity $\rho_{A}(t+d t), P_{A}(t+d t), \rho(t+d t)$ at the next time $\mathrm{t}+\mathrm{dt}$, And convert the density parameter to the pressure parameter $P(t+d t)$.

Continue to iterate to get the pressure value of the high-pressure fuel pipe at each moment:

$$
P(0), P(0+d t), P(0+2 d t), \cdots, P(T)
$$

Calculate the sum of the deviations between the pressure value and the rated value at these moments (objective function):

$$
S\left(T_{A}\right)=\sum_{i=1}\left|P(0+i d t)-P_{e}\right| d t
$$

Step 3 Variable decision variable $\omega$, Find the minimum value of the deviation $\mathrm{S}$, at this time the corresponding $\omega^{*}$ is the optimal angular velocity, namely:

$$
S\left(\omega^{*}\right)=\operatorname{Min}_{\omega} S\left(T_{A}\right)
$$

Using the mathematical software Matlab to program the solution model, and use the solution data to make the angular velocity-error relationship image, as shown in Figure 7.

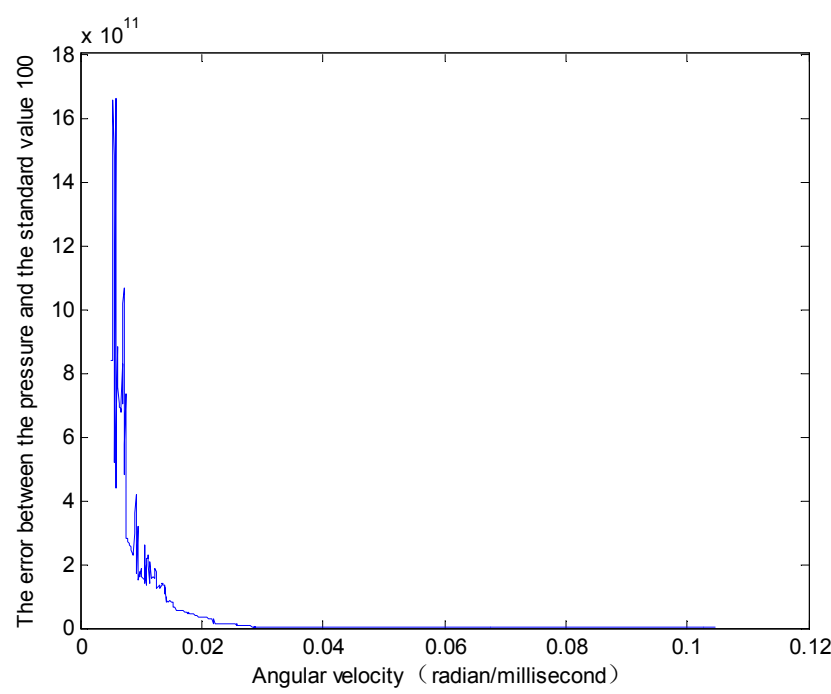

Figure 7. The relationship between angular velocity and error of cam.

It can be seen from the figure that the higher the speed of the cam, the smaller the total deviation of the pressure in the high-pressure fuel pipe from the standard value. However, the cam speed is generally between $100 \mathrm{rpm}$ and $200 \mathrm{rpm}$, that is, between $0.01047 \mathrm{radians} / \mathrm{ms}$ and $0.02094 \mathrm{radians} / \mathrm{ms}$. In order to minimize the error, blindly increasing the speed is not worth the gain, and too high speed is difficult to achieve in mechanical design. Therefore, we choose the optimal speed $\omega^{*}$ as the angular velocity corresponding to the inflection point in the figure above. Its meaning is: before $\omega^{*}$, as the speed increases, the error decreases rapidly; after $\omega^{*}$, as the speed increases, the rate of error decrease becomes slower. It can be seen from the figure that the optimal value is between 0.02-0.03. After programming numerical calculation, the optimal angular velocity value $\omega^{*}=0.0220$ radians $/ \mathrm{ms}$, that is, 210.0845 revolutions/minute is obtained. 


\subsection{Model Solution in the Case of Question 3}

The solution of problem 3 is similar to that of problem 2, only minor adjustments are needed. Under given new conditions, we find the corresponding parameters of the model, and add several items to the corresponding iterative formula, then we can substitute into the optimization model to solve.

\subsubsection{Add Another Optimization Model in the Case of Injectors}

After adding an injector, the iterative law of fuel parameters in the plunger cavity remains unchanged; and the iterative relationship of fuel density in the high-pressure fuel pipe needs to add an item corresponding to the new injector:

$$
\begin{aligned}
& \rho(t+d t)= \\
& \rho(t)+\frac{x_{\text {in }}(t) \rho_{A}(t) Q_{A}(t) d t-x_{\text {out }}(t) \rho(t) Q_{B}(t) d t-x_{\text {out } 2}(t) \rho(t) Q_{B}(t) d t}{V}
\end{aligned}
$$

Where, $x_{\text {out } 2}(t)$ is the injection characteristic function of the newly added injector.

If two injectors are allowed to work completely synchronously, $x_{\text {out } 2}(t)=x_{\text {out }}(t)$, the model at this time is equivalent to double the cross-sectional area of injector B in question 2.

In order to increase the flexibility of the model, the two injectors can be staggered to work, this only needs to make

$$
x_{\text {out } 2}(t)=x_{\text {out }}(t)\left(t+T_{\text {alpha }}\right)
$$

Where, Talpha represents the time difference between the working hours of the new fuel injector and the original fuel injector.

At this time, the objective function remains unchanged and remains

$$
\operatorname{Min} S\left(\omega, T_{\alpha}\right)=\sum_{i=1}\left|P(0+i d t)-P_{e}\right| d t
$$

Constraints are still given in actual meaning: $\omega \in$ $(0,0.1), T_{\alpha} \in[0,100]$.

The decision variables are the angular velocity $\omega$ of cam rotation and the working time difference $T_{\alpha}$ of the two injectors.

Solving this optimization model, you can get the best parameters $\omega$ and $T_{\alpha}$, that is, get the best fuel injection strategy and fuel supply strategy. Since a one-way pressure reducing valve is added to the next question, we regard the following situation as a broader situation. We will not solve the optimization model for this situation for the time being. Instead, we will solve the model in the more general case in Section 3.3.2 below, and then take the special case and return to the model in Section 3.3.1 to obtain the solution of the model in this section.

\subsubsection{The Optimization Model in the Case of Adding a Fuel Injector and a One-Way Pressure Reducing Valve}

After adding a fuel injector and a one-way pressure reducing valve, the iterative law of fuel parameters in the plunger cavity remains unchanged; while the iterative relationship of fuel density in the high-pressure fuel pipe needs to add one more one-way pressure reducing valve:

$$
\rho(t+d t)=\rho(t)+\frac{x_{\text {in }}(t) \rho_{A}(t) Q_{A}(t) d t-\left[x_{\text {out }}(t)+x_{\text {out } 2}(t)\right] \rho(t) Q_{B}(t) d t-x_{\text {out } 3}(t) \rho(t) Q_{D}(t) d t}{V}
$$

Where $x_{\text {out } 3}(t)$ is the fuel injection characteristic function of the newly added fuel injector, and its opening time is recorded as $T_{D}$. For convenience, it is still assumed that the pressure reducing valve needs to be closed every time it is opened $T_{D}{ }^{\prime}$ milliseconds, then

$$
x_{\text {out } 3}(t)=\left\{\begin{array}{c}
1,0 \leq t<T_{D} \\
0, T_{D} \leq t \leq T_{D}+T_{D}{ }^{\prime} \\
x_{\text {out } 3}\left[t-\left(T_{D}+T_{D}{ }^{\prime}\right)\right], t>T_{D}+T_{D}{ }^{\prime}
\end{array}\right.
$$

The flow rate $Q_{D}(t)$ is still determined by the flow rate formula:

$$
Q_{D}(t)=C A_{D} \sqrt{\frac{2 \Delta P}{\rho(t)}}=C A_{D} \sqrt{\frac{2[P(t)-0.5]}{P(t)}}
$$

Because the diameter of the pressure reducing valve $d_{D}=1.4$ has been given, the cross-sectional area $A_{D}=1.5394$ can be obtained.

At this time, the objective function remains unchanged and remains,

$$
\operatorname{Min} S\left(\omega, T_{\alpha}, T_{D}, T_{D}{ }^{\prime}\right)=\sum_{i=1}\left|P(0+i d t)-P_{e}\right| d \mathrm{t} .
$$

Constraints are still given in actual meaning: $\omega \in$ $(0,0.1), T_{\alpha} \in[0,100], T_{D}$ and $T_{D}{ }^{\prime} \in[0,100]$.
The decision variables are $\omega, T_{\alpha}, T_{D}, T_{D}{ }^{\prime}$.

In the numerical solution process, in order to facilitate the solution and mechanical design, we assume that the two injectors not only have the same structure, but also work synchronously, with the instant difference $T_{\alpha}=0$. Since the pressure reducing valve $\mathrm{D}$ has the same size parameters as the one-way valve $\mathrm{A}$ in question 1 , in order to simplify the solution of the model, it may be assumed that the performance of the two is exactly the same, that is, the one-way valve $\mathrm{D}$ must be closed for 10 milliseconds after one operation, which is a fixed parameter $T_{D}{ }^{\prime}=10$. Substituting specific data and solving the optimization model, the remaining two optimization parameters are obtained: $\omega=0.0312$, which is $297.9381 \mathrm{rpm}$, and $T_{D}=1$ millisecond.

In the model of this section, you only need to take $T_{D}=0$, that is, the pressure reducing valve will never open, and the situation in section 3.1 is obtained. Solving the optimization model obtains: the optimal speed of the cam $\omega^{*}=0.015$.

\section{Conclusion}

In this paper, we have explored the law of fuel pressure change in the high-pressure oil pipe. On one hand, we have used the calculus method to accurately describe the physical 
change law of fuel pressure. On the other hand, we have proposed the discrete algorithm to carry out the numerical simulation. The numerical results have shown the effectiveness of our mathematical model. In the future, we will improve the existing mathematical model to deal with more complex oil supply system.

\section{Fund Project}

The project of Western national social science fund (18XTJ002); Young and middle-aged teachers in colleges and universities of guangxi in 2019 basic ability to improve scientific research projects (2019KY0720).

\section{References}

[1] www.mcm.edu.cn. CUMCM 2019 Problems [EB/OL]. http://www.mcm.edu.cn/html cn/node/b0ae8510b9ec0cc0deb 2266d2de19ecb.html, 2019. 09 .

[2] Ahmed Jabbar Hussein, Hashim A. Kashkool. A weak Galerkin finite element method for two-dimensional coupled burgers' equation by using polynomials of order $(\mathrm{k}, \mathrm{k}-1, \mathrm{k}-$ 1) $[\mathrm{J}]$. Journal of Interdisciplinary Mathematics, 23 (4): 777-790.

[3] Hu Ying; Yang Jianguo; Hu Nao. Experimental study and optimization in the layouts and the structure of the high-pressure common-rail fuel injection system for a marine diesel engine $[\mathrm{J}]$. International Journal of Engine Research, 2021, 22 (6): 1850-1871.
[4] Zhong Qi, He Xianjian, LI Yanbiao. Research on Control Algorithm for High-speed on/off Valves that Adaptive to Supply Pressure Changes [J]. Journal of Mechanical Engineering, 2021, 57 (3): 224-235.

[5] Yang Yong-qi, Xu Xin-lei, Xu Hai-yang. Research on High Pressure Oil Pipe Pressure Control Based on Multi-Objective Dynamic Programming Model [J]. Computer Knowledge and Technology, 2020, 16 (6): 25-26.

[6] Mehdi AbiarKashani; Younes Alizadeh Vaghasloo; Mostafa AghaMirsalim. Optimal design of high-pressure fuel pipe based on vibration response and strength using multi-objective genetic algorithm [J]. Structural and Multidisciplinary Optimization, 2021, 1-22.

[7] Zajac Roman, Prokop Aleš, Řehák Kamil. Modal properties diagnostics of the high-pressure fuel injection pipes in off-road diesel engine [J]. Journal of Measurements in Engineering, 2021, 9 (01): 48-57.

[8] Xu Yuanzhen, Fang Yi, Sun Sirong. High-pressure Fuel Pipe Pressure Control Strategy Based on Multiple Search Algorithms [J]. Mathematical Modeling and Its Applications, 2020, 9 (4): 110-115.

[9] Wang Jianling, Yang Xiuqin. Elastic modulus of gas spring [J]. Journal of Pingdingshan Institute of Technology, 2005, 14 (2): 65-66.

[10] Shao Shiquan, Liu Xia. Discussion and study on the equation form of cam profile curve [J]. Mechanical Research and Application, 2014, (3): 1-2. 